\title{
Cross-Cultural Adaptation and Psychometric Properties of an Arabic Version of the Western Ontario Shoulder Instability Index (WOSI)
}

\author{
Dr Aliaa Khaja ${ }^{* 1}$; Dr Ahmed Bouhamra ${ }^{2}$; Dr Sager Hanna ${ }^{3}$; Dr Ali Maqdis ${ }^{4}$ \\ ${ }^{1}$ Orthopedic Resident, Al Razi Orthopedic Hospital Kuwait. Email: aliaakhaja@moh.gov.kw \\ ${ }^{2}$ Sports Surgeon Specialist, Al Razi Orthopedic Hospital Kuwait. Email: ahmed.bouhamra@gmail.com \\ ${ }^{3}$ Orthopedic Resident, Al Razi Orthopedic Hospital Kuwait. Email: sager.s.hanna@gmail.com \\ ${ }^{4}$ Sports Surgeon Specialist, Al Razi Orthopedic Hospital Kuwait. Email: dralimaqdisi@gmail.com \\ *Corresponding author: Dr Aliaa Khaja; aliaa.khaja@googlemail.com
}

Received: 20 November 2020;

Accepted: 29 November 2020;

Published: 01 December 2020

\begin{abstract}
Background: The Western Ontario Shoulder Instability Index (WOSI) score is a tool that helps with self-assessment of the shoulder's functional status in patients experiencing instability problems. The purpose of this study was the cross-cultural adaptation of WOSI into Arabic and assessment of its psychometric properties in comparison to a gold standard-questionnaire, namely the Arabic Disability of the Arm, Shoulder and Hand (DASH) score. Material \& Methods: 100 patients participated in this survey, tested initially and retest after two months. The internal consistency tests were performed using Cronbach's alpha. Besides, Pearson's Correlation and Standard response mean (SRM) were calculated to estimate criterion validity and responsiveness of the Arabic WOSI in comparison to the Arabic DASH. Results: The Arabic WOSI had a Cronbach's alpha score of 0.85 at the baseline and 0.91 at the follow-up time period. All subscales had an internal consistency greater than 0.7, except Sport/Work (0.69 at follow-up). A strong correlation with Arabic DASH score was observed ( $\mathrm{r}=0.79$ at baseline \& 0.87 at Follow-up) which suggested good validity. Also, moderately correlated changes of baseline to follow-up in DASH and WOSI indicated moderate responsiveness. No ceiling and floor effects were observed among the responses. Conclusion: Overall, the Arabic version of WOSI proved to be a good and reliable diagnostic tool for patients with shoulder instability.
\end{abstract}

Keywords: Shoulder surgery, shoulder instability, shoulder score, patient-related outcome

\section{Introduction}

Quite a number of tools have been developed to help test the results of orthopedic treatment of shoulder defects, such as instability problems. Shoulder instability causes problems with assessment due to intermittent symptoms and less pain, rather than anticipation of the problems that may be caused by certain activities. There is a need for instruments that concentrate on the subjective viewpoint of the patient concerning the outcome. Kirkley et al. (1998) developed such a patient-specific scoring system for shoulder disorders ${ }^{[1]}$. He evaluated the score properties with the original English version - Western Ontario Shoulder Instability Index (WOSI), using established guidelines ${ }^{[2]}$.

The Western Ontario Stability Index may be administered via mail and is user-friendly. Its high sensitivity and intraclass correlation coefficient (ICC) shows that it can be used for monitoring the functional status (progress) of each patient. WOSI can as well be used for clinical trials and helps with comparison and aggregation of cohort studies. The objectives of patientevaluated outcome measures are supplementation of conventional outcome measures such as strength, range of motion, and other major symptoms ${ }^{[3]}$.

The major psychometric properties used in validating scores include responsiveness, reliability, and validity. Responsiveness refers to the ability of a particular score to measure changes with time. Reliability is the extent to which an outcome measure can be reproduced. Validity shows whether a particular instrument measures precisely what it is supposed to measure. The simplicity of the scoring system is also of utmost importance. It should be highly effective, and easy to use. This will encourage orthopedic surgeons to incorporate it into their practice.

Poolman et al. (2009), mentioned cultural and linguistic considerations for a specific population when attempting to translate and validate certain assessment tools ${ }^{[4]}$. This study aims to retest the Arabic version of WOSI score's psychometric properties. 


\section{Material \& Methods}

\section{Translation}

Translation was done as per recommendations of Guillemin's guidelines for validation and cross-cultural adaptation after permission obtained from the original WOSI copyright holder ${ }^{[2]}$. Two Bilingual orthopedic surgeons were responsible for the conceptual and literary translation of the original WOSI. Two other versions were produced by independent translation companies with a background in scientific English. All the versions produced were similar. Modifications to incorporate from all the versions were made and implemented in the final version. A professional Arabic grammar checker reviewed it. The back-translation came close to the original score.

\section{Participants}

100 patients participated in this study and completed the WOSI and DASH questionnaires. They agreed to have their data analysed for research purposes. The mean age of participants was 55.1 years. The age range of the participants was between $20-83$ years. It took the participants approximately 4 minutes to fill out the questionnaire. A pilot test was initially conducted on 7 random patients from the arthroplasty clinic, the investigators interviewed them after the filled out the Arabic questionnaire to inquire about ease of language and understanding of the content.

\section{Psychometric Properties and Data Analysis}

Presentation of the outcome measures of each construct was done using descriptive analysis. Mean and standard deviation (SD) were calculated. Internal consistency was evaluated by calculating the Cronbach's $\alpha$. According to the literature, $\alpha>0.70$ was regarded as acceptable, though it should not exceed 0.95 , to avoid redundancy [5].

Table 1: Descriptive analysis of Baseline and follow-up outcome measures

\begin{tabular}{|l|l|l|l|l|l|}
\hline & Baseline & & \multicolumn{2}{l|}{ Follow-up } \\
\hline & Mean & SD & & Mean & SD \\
\hline DASH & 55.2 & 18.5 & & 35.6 & 16.5 \\
\hline Total WOSI & 72.9 & 20.2 & & 50.4 & 18.4 \\
\hline Physical Symptoms & 62.3 & 18.2 & & 43.5 & 19.7 \\
\hline Sports/Recreation/Work & 85.4 & 15.3 & & 57.7 & 23.5 \\
\hline Lifestyle & 82.9 & 16.7 & 59.5 & 20.3 \\
\hline Emotions & 60.9 & 26.1 & 40.7 & 23.3 \\
\hline
\end{tabular}

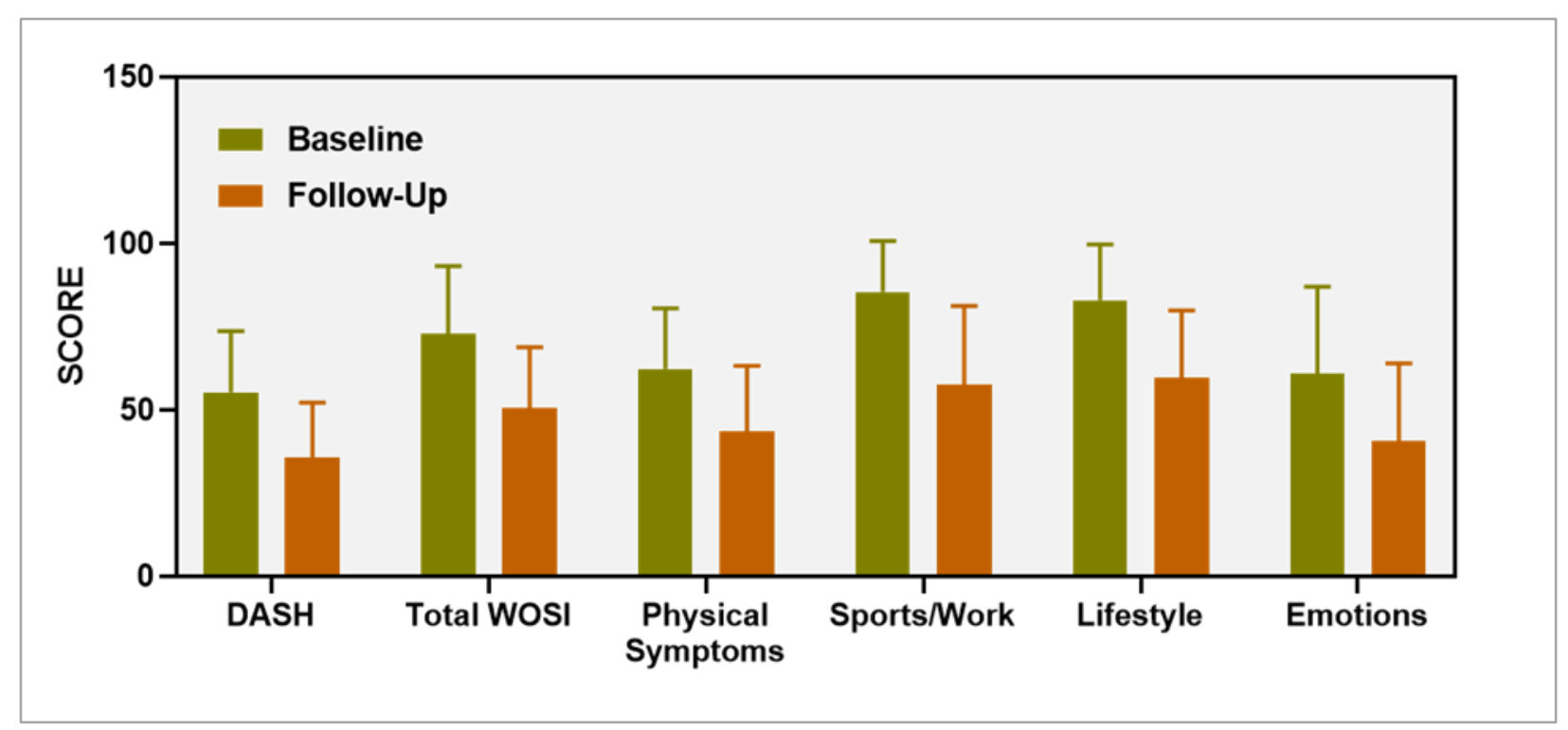

Figure 1: Baseline and follow-up of Arabic DASH, Total WOSI, and its subscales 


\section{Reliability}

To estimate the reliability of the questionnaire, Internal consistency was calculated by using overall Cronbach's alpha which was equal to 0.85 at the baseline and 0.91 at follow-up, indicating a high degree of internal consistency in both time frames. All subscales showed an acceptable degree of internal consistency expect sport/recreation/work, which in the Follow-up did not reach the minimum value of $0.70(\alpha=0.69)$

Table 2: Internal consistency of the Arabic version of WOSI in baseline and follow-up questionnaire

\begin{tabular}{|l|l|l|l|}
\hline & \multirow{2}{*}{$\begin{array}{l}\text { Number } \\
\text { of items }\end{array}$} & \multicolumn{2}{|l|}{ Cronbach's $\boldsymbol{\alpha}$} \\
\cline { 4 - 5 } & 21 & Baseline & Follow-Up \\
\hline Total WOSI & 10 & 0.85 & 0.91 \\
\hline Physical Symptoms & 4 & 0.75 & 0.75 \\
\hline Sports/Recreation/Work & 4 & 0.75 & 0.69 \\
\hline Lifestyle & 4 & 0.79 & 0.87 \\
\hline Emotions & 3 & \multicolumn{2}{|l}{} \\
\hline
\end{tabular}

\section{Responsiveness \& Criterion validity}

The Arabic versions of WOSI and DASH scores indicated a strong correlation both at baseline $(r=0.79, \mathrm{p}<0.001)$ and at follow-up ( $\mathrm{r}$ $=0.87, \mathrm{p}<0.05)$. This was an indication of strong criterion validity. The changes in WOSI and DASH from baseline to followup were proved to be moderately correlated $(\mathrm{r}=0.63, \mathrm{p}<0.001)$, this correlation could also be partly shown in Figure 1, where the trends of both questionnaires indicating fair responsiveness. Also, the SRM (Standard response mean) for both measures are calculated with WOSI SRM $=1.45$ and DASH SRM $=1.51$, which was quite large for both of them.

\section{Discussion}

We had a high test-retest reliability of WOSI, with a Cronbach's alpha of 0.85 and 0.91 at baseline and follow-up respectively. ICC values for the different items were ranging at 0.70 and 0.79 at the baseline, and $0.75-0.87$ at follow-up. According to Bot et al., the WOSI score can be applied herein as well as in the respective domains - except for the sports/recreation/work domains ${ }^{[7]}$. That fact that the internal consistency was 0.91 for the baseline WOSI compares well with the internal consistency of 0.95 in the original publication. This is a clear indication that there was no dramatic change in the score properties, hence encouraging the use of the Arabic Version of the WOSI.

Many studies have attempted translation and cross-cultural adaptaion of the WOSI into different languages throughout the world for use in their respective countries.

In 2009, a Swedish translation and validation by Salomonsson et al. was done ${ }^{[8]}$. The Cronbach alpha was 0.89 and 0.95 for pre-operative and post-operative respectively. The pearson coefficient was 0.59 , while their ICC was 0.94 . They however compared the WOSI vs the Rowe score ${ }^{[8]}$.

A year later, Drerup et al. presented their WOSI translated into the German language to be used for German speakers ${ }^{[9]}$. The Cronbach alpha for the German WOSI was 0.89 for the total score [9]. Their test-retest reliability (ICC) ranged from 0.73- 0.90, compared to our 0.70-0.79 at baseline for the Arabic WOSI.

The Japanese WOSI by Hatta et al. (2011) was done comparing the WOSI to the quick-DASH score as well as the Rowe Score ${ }^{[10]}$. The Cronbach alpha (0.84) was similar to our Arabic WOSI while the Pearson's coefficient between the Japanese
WOSI and quick-DASH score was 0.63 , as opposed to our $0.79^{[10]}$. They concluded in their study that the Japanese WOSI is a reliable self-assessment tool that is comparable to the original WOSI ${ }^{[10]}$.

Cacchio et al. translated the WOSI into Italian and their study showed a Cronbach alpha of 0.93 , ICC of 0.95 at baseline and 0.92 on follow up ${ }^{[11]}$. Their construct validity correlated more between the DASH (0.79) than with the SF-36 (0.11) ${ }^{[11]}$.

In 2014, a Dutch translation and validation of the WOSI score and its correlation between the DASH score was done ${ }^{[12]}$. The total score Cronbach alpha was 0.96 while the total ICC was 0.92 (range from $0.88-0.920^{[12]}$. There was also a high correlation with the DASH score at $0.81^{[12]}$.

All 100 patients involved in the study experienced improvements in the WOSI score, and this agrees with the standardized response mean (SRM) of 1.45 for WOSI and 1.51 for DASH, both being larger than the SRM in the original presentation (which was 0.93) ${ }^{[1]}$. A French WOSI was translated, validated, and responsiveness checked against the quick-DASH as well, a study by Guadelli et al. $(n=144)^{[13]}$. In their study, the SRM was 1.55 (French WOSI) and 0.87 (Quick DASH) ${ }^{[13]}$. These corresponded to a strong correlation $(>0.80)$. The SRM is an effective size index, obtained by dividing the mean change in scores by the standard deviation of the score, and is one of the best measures to estimate responsiveness ${ }^{[13]}$.

The significance of the WOSI in being a reliable and the patient-related outcome measure has led to the multiple translations and validation studies have been shown to be reliable in their respective languages and recommended for use with patients of different languages. In the International Society of Arthroscopy, Knee Surgery and Orthopaedic Sports Medicine.

(ISAKOS) Scientific Committee Report comparing different scoring systems for the functional Assessment of the Shoulder, the WOSI was more responsive than other tools for Shoulder Instability ${ }^{[14]}$.

It is worth noting that conventional clinician-based parameters like strength and motion do not directly evaluate shoulder functions, which is necessary for the assessment of prognosis. An ideal scoring system should be targeted at the functional outcome, and it is necessary to prioritize the patient's point of view ${ }^{[15]}$. This score evaluation indicates that the score assesses important symptoms and not just stability.

\section{Conclusion}

The retest showed the Arabic version of the WOSI score having a highly reliable consistency and good validity with high responsiveness, all at a similar level as in the original score. We suggest that WOSI be employed in the evaluation of patients experiencing issues with instability.

\section{Abbreviations}

WOSI- Western Ontario Shoulder Instability Index

DASH- Disability of the Arm, Shoulder and Hand Score

SRM- Standard response mean

ICC- intraclass correlation coefficient

SD- standard deviation

ISAKOS- International Society of Arthroscopy, Knee Surgery and Orthopaedic Sports Medicine

DECLARATIONS 


\section{Ethical approval and Consent for publication}

- $\quad$ Ethical approval was obtained

- Consent of publication was obtained in writing from all participants

- Name of Ethical Committee: Ministry of Health, Kuwait, Research and publication office

- Committee Reference Number: 1061/2019

\section{Consent to publish}

Consent of participation and publish was obtain with written Format from all participants.

\section{Availability of data and material}

The data that support the findings of this study are available from Ministry of Health Al Razi Orthopedic Hospital, Kuwait. However, restrictions apply to the availability of these data, which were used under license for the current study, and so are not publicly available. Data is available from the authors upon reasonable request and with permission of Ministry of Health $\mathrm{Al}$ Razi Orthopedic Hospital, Kuwait.

\section{Competing interests}

The authors declare that they have no competing interests.

\section{Funding}

No funding was supplied in this study.

\section{Authors' Contributions}

Dr. Ahmed Bouhamra: He had the task of doing a literature review of similar topic that have relevance to our study. He had a role in data collection and writing the abstract first draft of the manuscript.

Dr Aliaa Khaja: Methodology, formal analysis and project administration was her responsibility. She made sure the data was complete and usable. The first draft of the manuscript was written by her as well.

Dr. Sager Hanna: Revised the final draft of the manuscript as well as conducted the investigation and data collection to ensure there was an adequate sample size for the study.

Dr. Ali Maqdisi: He formulated the topic in question for this study. As such, he also held the role of supervisor and set the aims and goals for this study. Team members went back to him for mentorship.

\section{Acknowledgment}

The original authors of WOSI gave their permission, we thank them very much - they were involved in all stages etc.

\section{References}

[1] Kirkley A, Griffin S, Mclintock H, Ng L. The development and evaluation of a disease-specific quality of life measurement tool for shoulder instability. The Western Ontario Shoulder Instability Index (WOSI). Am J Sports Med. 1998;26(6):764-72.
[2] Guillemin F, Bombardier C, Beaton D. Cross-cultural adaptation of health-related quality of life measures: literature review and proposed guidelines. J Clin Epidemiol. 1993;46(12):1417-32.

[3] Lillkrona U. How should we use the Constant Score? - A commentary. J Shoulder Elbow Surg. 2008;17((2)):3623.

[4] Poolman, R. W., Swiontkowski, M. F., Fairbank, J. C., Schemitsch, E. H., Sprague, S., \& de Vet, H. C. (2009). Outcome Instruments: Rationale for Their Use. The Journal of Bone and Joint Surgery-American Volume, 91(Suppl 3), 41-49. doi:10.2106/jbjs.h.01551.

[5] Fayers PM, Machin D. Quality of life: the assessment, analysis and interpretation of patient-reported outcomes. Manhattan: John Wiley \& Sons; 2013.

[6] Terwee, Caroline \& Bot, Sandra \& Boer, Michiel \& van der Windt, Danielle \& Knol, Dirk \& Dekker, Joost \& Bouter, Lex \& De Vet, Henrica. (2007). Quality criteria were proposed for measurement properties of health status questionnaires. Journal of clinical epidemiology. 60. 34-42. 10.1016/j.jclinepi.2006.03.012.

[7] Bot SD, Terwee CB, van der Windt DA, Bouter LM, Dekker J, de Vet HC. Clinimetric evaluation of shoulder disability questionnaires: a systematic review of the literature. Ann Rheum Dis. 2004;63((4)):335-41.

[8] Salomonsson B, Ahlstrom S, Dalen N, Lillkrona U. The Western Ontario Shoulder Instability Index (WOSI): validity, reliability, and responsiveness retested with a Swedish translation. Acta Orthop. 2009;80:233-238.

[9] Drerup S, Angst F, Griffin S, Flury MP, Simmen BR, Goldhahn J. Western Ontario shoulder instability index (WOSI): translation and cross-cultural adaptation for use by German speakers. Orthopade. 2010;39:711-718.

[10] Hatta T, Shinozaki N, Omi R, Sano H, Yamamoto N, Ando A, Sugaya H, Aizawa T, Kuriyama S, Itoi E. Reliability and validity of the Western Ontario Shoulder Instability Index (WOSI) in the Japanese population. J Orthop Sci. 2011;16:732-736.

[11] Cacchio A, Paoloni M, Griffin SH, Rosa F, Properzi G, Padua L, Padua R, Carnelli F, Calvisi V, Santilli V. Cross-cultural adaptation and measurement properties of an Italian version of the Western Ontario Shoulder Instability Index (WOSI) J Orthop Sports Phys Ther. 2012;42:559-567.

[12] Van der Linde, J.A., Willems, W.J., van Kampen, D.A. et al. Measurement properties of the Western Ontario Shoulder Instability Index in Dutch patients with shoulder instability. BMC Musculoskelet Disord 15, 211 (2014).

[13] Gaudelli, C., Balg, F., Godbout, V., Pelet, S., Djahangiri, A., Griffin, S., \& Rouleau, D. M. (2014). Validity, reliability and responsiveness of the French language translation of the Western Ontario Shoulder Instability Index (WOSI). Orthopaedics \& Traumatology: Surgery \& Research, 100(1), 99-103. doi:10.1016/j.otsr.2013.09.007.

[14] Kirkley A, Griffin S, Dainty K. Scoring systems for the functional assessment of the shoulder. Arthroscopy. 2003;19(10):1109-20.

[15] Romeo AA, Bach BR, Jr, O'HALLORAN KL. Scoring systems for shoulder conditions. Am J Sports Med. 1996;24(4):472-6. 